\title{
Male-to-Female (MtoF) gender affirming surgery: Modified surgical approach for the glans reconfiguration in the neoclitoris (M-shape neoclitorolabioplasty)
}

\author{
Andrea Cocci ${ }^{1}$, Francesco Rosi ${ }^{2}$, Davide Frediani ${ }^{2}$, Michele Rizzo ${ }^{3}$, Gianmartin Cito ${ }^{1}$, Carlo Trombetta ${ }^{3}$, \\ Francesca Vedovo ${ }^{3}$, Simone Grisanti Caroassai ${ }^{1}$, Augusto Delle Rose ${ }^{1}$, Valeria Matteucci ${ }^{4}$, \\ Piero Buccianti ${ }^{4}$, Cristina Ceccarelli ${ }^{4}$, Marco Carini ${ }^{1}$, Andrea Minervini ${ }^{1}$, Girolamo Morelli ${ }^{2}$ \\ ${ }^{1}$ Careggi Hospital, Department of Urology, University of Florence, Florence, Italy; \\ ${ }^{2}$ Department of Urology, University of Pisa, Pisa, Italy; \\ ${ }^{3}$ Department of Urology, University of Trieste, Trieste, Italy; \\ ${ }^{4}$ Department of General Surgery, University of Pisa, Pisa, Italy.
}

\begin{abstract}
Summary Purpose: The aim of this article is to describe our modified surgical technique for the reconfiguration of the glans in the clitoris and the labia minora, known as the "M-shape neoclitorolabioplasty".

Methods: The glans with all its neurovascular bundle is isolated from the corpora cavernosa, incised in Y-shape mode and spread in order to obtain an M-shape glandular flap. The "belly" of the M-shape glans will constitute the triangular neoclitoris meanwhile the lateral flaps will constitute the labia minora. The inferior apex of the neoclitoris is fixed to the superior apex of the previously spatulated urethra. The two glans flaps are incised transversally to increase their length and sutured to the sides of the spatulated urethra forming the labia minora. Our technique permits to create an aesthetically pleasing neovagina preserving all the glandular erogenous sensitivity.

Results: 94 patients have been treated with our modified technique of male-to-female (MtoF) gender affirming surgery. At median follow-up of 27.57 months, 81 (86.1\%) patients reported vaginal intercourse and $78(82.9 \%)$ patients referred presence of erogenous sensitivity during dilatations, intercourse or masturbations. All the glandular tissue is preserved and reconfigured forming the neoclitoris and the labia minora. The M-shape reconfiguration permit to create an aesthetically pleasant neoclitoris.

Conclusions: This technique could be applied safely and easily to patients undergoing gender affirming surgery, allowing the creation of a neovagina with the best possible erogenous sensitivity without losing aesthetical results.
\end{abstract}

KEY WORDS: Male to female; Gender affirming surgery; Transgender/transsexual.

Submitted 15 January 2019; Accepted 26 January 2019

\section{INTRODUCTION}

Gender Dysphoria (GD) and Gender Identity Disorder have been defined in the Diagnostic and Statistical Manual of Mental Disorders, Fifth Edition (1) and in the International Classification of Diseases, Tenth Edition (2). According to the Standards of Care of the World Professional Association of Transgender Health (WPATH) (3), the management of patients presenting with Male-to-Female (MtoF) GD is complex and multifactorial. The surgical treatment of GD is the gender affirming surgery. In MtoF patients, gender affirming surgery involves the creation of a neovagina (vaginoplasty) and the reconstruction of a sensate neoclitoris (neoclitoroplasty) from the penile glans. During the years we have perfected the neoclitorolabioplasty with the specific goal of preserving as much erogenous tissue as possible in order to achieve best possible functional and aesthetic outcomes. The aim of this paper is to illustrate our gender affirming surgery technique focusing on the M-shape neoclitorolabioplasty and its outcomes.

\section{Materials AND METHOdS}

Indications for procedure

Transsexual women are sent to MtoF gender affirming surgery according to the seventh version of the WPATH (3) standard of care.

Furthermore, according to Italian laws, all patients have to obtain a formal court authorization for this specific procedure.

\section{Preoperative preparation}

All the patients interrupt Progynova ${ }^{\circledR}$ (estradiol valerate) 30 days before gender affirming surgery (for thromboembolic risk) and start it again 15 days after gender affirming surgery. A low-residue diet and bowel preparation are recommended to avoid postoperative contamination of the surgical wound. Metronidazole and amoxicillin clavulanate are given routinely as prophylactic antibiotics. Patients are placed in lithotomy position and intermittent pneumatic compression is used to prevent deep venous thrombosis. The procedure is performed under General anesthesia. A urethral catheter is placed to locate the urethra during the entire procedure. The surgical area is sterilized with Betadine ${ }^{\circledR}$ (povidone-iodine) solution. The standard equipe included two surgical teams each composed by two surgeons who perform this surgery. Team A operates between the legs of the patient and team $B$ is positioned at the level of the abdomen.

No conflict of interest declared. 


\section{Intraoperative considerations}

The surgery is divided in three main steps:

A) Management of the perineal space, performed by team A:

1. Castration (orchiectomy)

2. Creation of the neovaginal cavity;

3. Excision of the crura;

4. Reduction of the bulb of penis, preparation of urethral stump and spatulating of it;

B) Management of the penis, performed by team B:

5. Degloving of the penis, dissection of the gland and its neurovascular bundle;

6. Dissection of the pendulous urethra and excision of the corpora cavernosa;

7. Creation of the neoclitoris and the labia minora (neoclitorolabioplasty);

C) Management of the reconstruction of the external genitalia, performed by the two teams:

8. Creation of the labia majora and preparation of the cutaneous grafts;

9. Creation of the vulval vestibule, the clitoral hood and the urethral neo-meatus;

10. Creation of the cul-de-sac;

11. Introflection of the cul-de-sac and suture of the labia majora.

Step A) and B) are performed at the same time by the two teams working independently. Step C) is performed when the other two steps are completed.

A) Management of the perineal space, performed by team A 1. Castration (orchiectomy)

Initially, a racket shape marking is drawn for the incision in the perineal foreskin by a dermographic pen. The incision is performed along the medial raphe from the scrotum up to the perineum. The perineal skin flap, thus obtained, is inverted downwards, maintaining subcutaneous fat and preserving its vascularization. The testes and the spermatic cords are ligated and dissected at the level of the external inguinal rings, which are closed with a 2/0 Monocryl ${ }^{\circledR}$ continuous suture to prevent postoperative hernia formation. The peri-testicular fat is conserved for the secondary purpose of filling and shaping of the labia majora.

\section{Creation of the neovaginal cavity}

Undoubtedly this is the most complicated and risky step of the whole gender affirming surgery. Injuring of the rectum or the membranous urethra are possible complications. Fibers of bulbocavernous muscles are divaricated on the midline line and the bulbar urethra is completely freed. The bulbocavernous muscles are totally removed. The perineum is dissected creating a neo-cavity between the urethra and anus. Superficial and deep transverse perineal muscles are divaricated laterally.

The inferior fascia of urogenital diaphragm is exposed and cut laterally. After that a bluntly dissection is performed in order to create the neovaginal cavity. The dissection proceeds from the medial part of ischial tuberosity until the ischiorectal fossa through the endopelvic fascia. At the end of these procedure membranous urethra and the apex of prostate are reached. Then neovaginal cavity is enlarged detaching tissue between the urethra, the prostate, the urinary bladder anteriorly and the rectum posteriorly, up to the peritoneal reflection of the rectovesical pouch. The obtained depth is measured with a centimetered vaginal stent, which must be at least $12 \mathrm{~cm}$.

\section{Excision of the crura}

Meanwhile the penile urethra is bluntly dissected from the corpora cavernosa by team B, team A excises both the crura from their respective ischiopubic ramus. Remnants of spongiosus tissue are sutured with semicontinuous in Monocryl ${ }^{\circledR}$ 3/0.

4. Reduction of the bulb of penis, preparation of the urethral stump and spatulating of it

The bulb of penis is completely excised in order to increase the space of the vulvar introit. At this point the distal urethra is excised and the residual stump is ventrally spatulated and cut to create a lance-like shape of the urethral plate. The apex is fixed to the anterior portion of pubis with a single Monocry ${ }^{\circledR}$ 3/0 stitch. The spatulated urethral plate are sutured with semicontinuous in Monocryl ${ }^{\circledR} 3 / 0$ at both sides to the ischial branches.

With this urethral reconfiguration the anterior part of the neovagina until the neoclitoris is provided by urethral mucosa.

B) Management of the penis, performed by team $B$

5. Degloving of the penis, dissection of the glans and its neurovascular bundle

Team B starts with the degloving of the penis. A circumferential subcoronal incision is made and a cylindrical penile skin flap is created, including the foreskin in uncircumcised patients. The penile skin is bluntly dissected from the albuginea. The degloved penis is passed through the posterior scrotal window to facilitate the disassembly. The penile skin flap will constitute part of the wall of the neovagina. After clamping the penis at its base, the dorsal part of the glans along with its neurovascular bundle is bluntly dissected from the corpora cavernosa.

6. Dissection of the pendulous urethra and excision of the corpora cavernosa

The corpora cavernosa are bluntly dissected from the pendulous urethra (then cut and spatulated by team A), and excised at the levels of the pubis attachment.

7. Creation of the neoclitoris and the labia minora (neoclitorolabioplasty)

A Y-shape marking is drawn for the incision on the dorsal glans by a dermographic pen. The Y-shape incision is performed using the electro-cautery and following the markings. In this way an M-shape glans is obtained when it is spread. The "belly" of the M-shape glans will constitute the triangular neoclitoris meanwhile the lateral flaps will constitute the labia minora (Figures 1,2). During this step the neurovascular bundle is folded up on itself and the reflected side is fixed with three 3/0 Monocryl ${ }^{\circledR}$ simple stitches to the soprapubic region thus forming the mons Veneris. The inferior apex of the neoclitoris is fixed with a $3 / 0$ Monocryl ${ }^{\circledR}$ simple stitch to the superior apex of the spatulated urethra. The two glans flaps are incised transversally so that their length is increased and sutured 

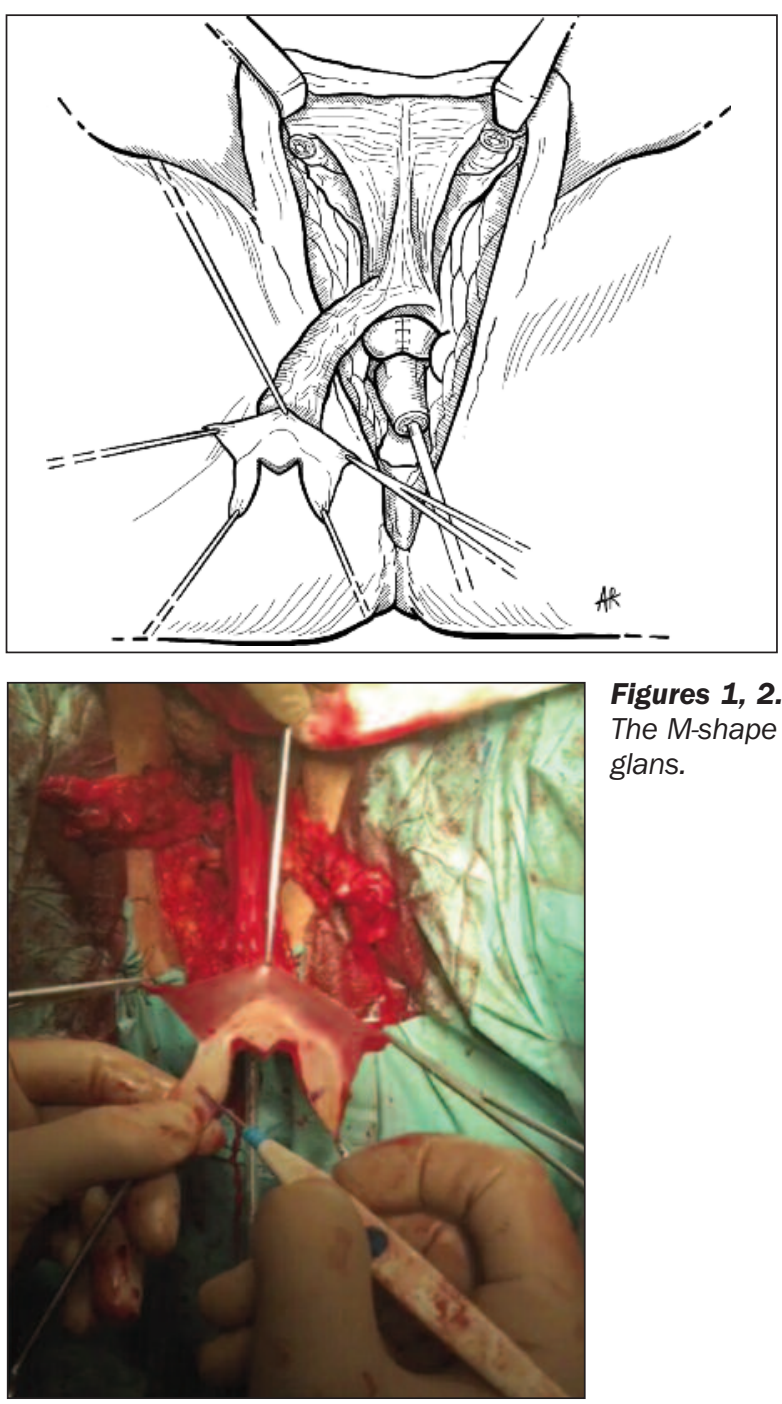

Figures 1, 2 The M-shape glans.

with $3 / 0$ Monocryl ${ }^{\circledR}$ to the sides of the spatulated urethra thus forming the labia minora.

C) Management of the reconstruction of the external genitalia, performed by the two teams

8. Creation of the labia majora and preparation of the cutaneous grafts

The peri-testicular fat (previously conserved by team A) is sutured with 2/0 Monocryl ${ }^{\circledR}$ to the inferior apex of perineum. The purpose of this action is to give volume and thickness to the labia majora. The latero-inferior portions of the penile skin flap are fixed with $2 / 0$ Prolene ${ }^{\circledR}$ simple stitches to the lower sides of the racket shape incision (initially made by team A). In this manner the inferior apex of the labia majora is formed. The redundant skin is drawn by dermographic pen and excised by electro-cautery. It will be used as cutaneous graft. On the surgical bench the two cutaneous grafts are thinned out of the subcutaneous fat and sutured together with 2/0 Monocryl ${ }^{\circledR}$.

9. Creation of the vulval vestibule, the clitoral hood and the urethral neo-meatus

At this point team B has finished its job and team A completes the surgery. The cylindrical penile skin flap is

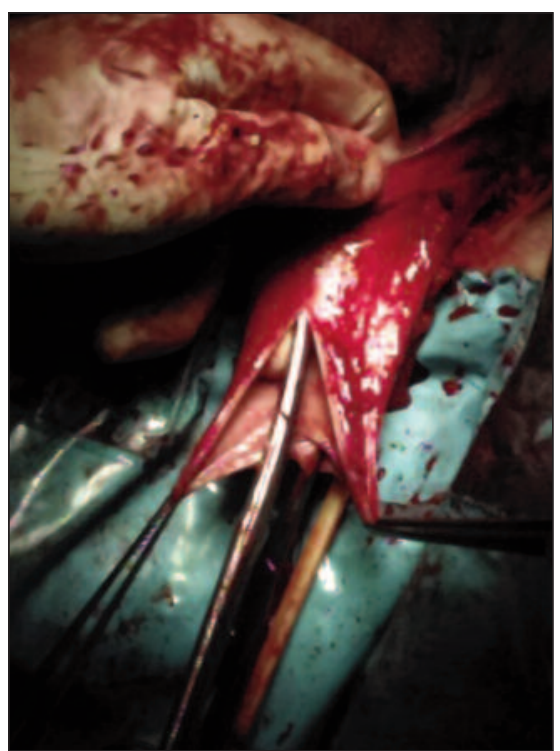

Figure 3.

Creation

of the clitoral

hood and

the labia majora.

reversed like a glove finger. Then the cutaneous base of it is stretched downwards. In this manner the skin covers sequentially the folded neurovascular bundle (forming the mons veneris), the neoclitoris, the labia minora and the spatulated urethra. The cutaneous base is fixed laterally and internally to the entrance of the neovaginal cavity with 2/0 Monocryl ${ }^{\circledR}$ simple stitches. Afterwards a longitudinal incision is made with scalpel on the covering skin paying attention not to cut the underlying structures. This action is made in order to evert the neclitoris, the labia minora and the spatulated urethra. The edges of the incision are spread. The anterior apex of the incision is sutured with $2 / 0$ Monocryl ${ }^{\circledR}$ to the top of neoclitoris forming the clitoral hood (Figure 3). The lateral sides are sutured to the labia minora. The posterior apex is sutured to the overturned urethral mucosa forming the urethral neo-meatus. Once these actions are completed, the vulval vestibule is obtained. In this manner the mucosa of the spatuleted urethra is used for the creation of the vulval vestibule so that the sensitive surface is increased.

\section{Creation of the cul-de-sac}

The vaginal stent is placed in the neovaginal cavity. The part that comes out of the cavity is used as a model to create the cul-de-sac. The penile skin flap is put on the vaginal stent and sutured with continuous in Monocryl ${ }^{\circledR}$ 3/0 to the perineal skin flap and the cutaneous grafts in order to obtain the cul-de-sac (Figure 4).

11. Introflection of the cul-de-sac and suture of the labia majora

The vaginal stent is removed and the cul-de-sac is turned inside out and pulled into the neovaginal cavity to become the wall of the neovagina. The labia majora are closed with 2/0 Monocryl ${ }^{\circledR}$ running intradermal suture in order to obtain the best aesthetical outcome (Figure 5).

\section{Dressing}

Once the surgery is completed, five gauzes impregnated with Betadine ${ }^{\circledR}$ and Gentalyn ${ }^{\circledR}$ are sewed sequentially and inserted in the neovaginal cavity. A tie-over dressing is 


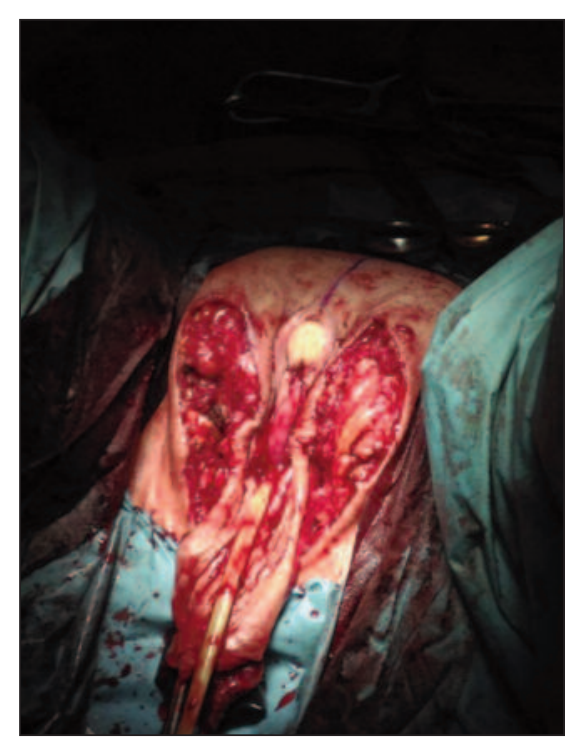

Figure 4.

Creation of the cul-de-sac.

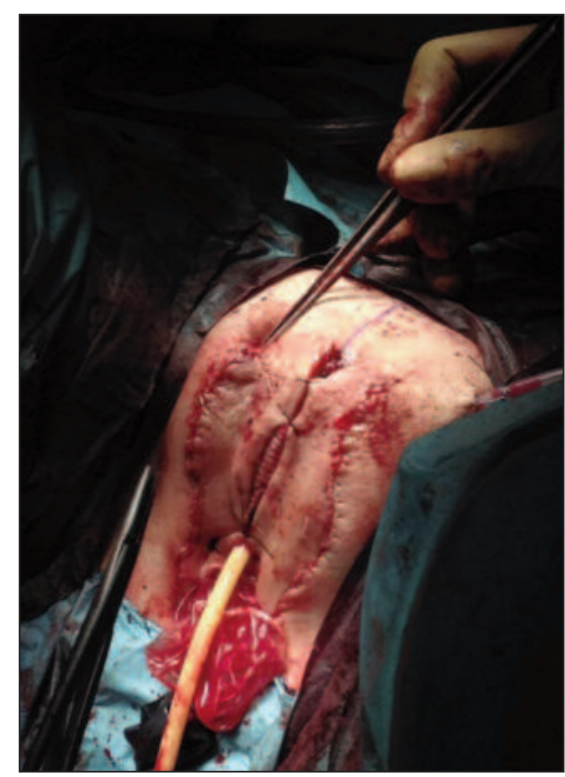

Figure 5.

Suture of labia majora.

then performed around the neo-meatal orifice. It is fixed with a Nylon skin suture. A customized elastic compressive dressing is applied to prevent bleeding.

\section{Postoperative management and follow-up}

During all the postoperative in-stay antibiotic prophylaxis with amoxicillin clavulanate $1 \mathrm{~g}$ per 3 days and Clexane $^{\circledR}$ (enoxaparin sodium) is given. Patients are encouraged to mobilize 24 hours after the surgery. They sit with the help of a donut cushion avoiding pressures on the neovagina. The vaginal intruder, the tie-over dressing, the compressive dressing and the urinary catheter are removed 4 days after the surgery and patients are trained to correctly perform daily vaginal dilatation two times per day for 30 minutes each. They are advised to clean the vagina daily with $1 / 3$ Betadine $^{\circledR}$ and 2/3 Saline solution through a $60 \mathrm{cc}$ cone-catheter syringe before and after the dilations. At the end of dilation, a dressing with gauzes and Gentalyn ${ }^{\circledR}$ is applied above the site of surgery. Patients are usually discharged within 1 week. Progynova ${ }^{\circledR}$ is restarted 15 days after the intervention. The vaginal dilations prosecute for 2 weeks with the same protocol. Then dilations are mandatory 3 times per day increasing gradually the diameter of the dilators according to individual tolerance. Dilations are continued at least for 2 months after the operation until the patients have regular sexual intercourse. Follow-up visits are scheduled at 15,30 and 60 days after the intervention, then every 3 months. At each visit, physical examination to assess congruous vaginal length and width, neoclitoris and labia minora sensation, cosmetic appearance of the reconstructed vagina and occurrence of any complication are performed. At 60 days visit postoperative urodynamic studies are also performed.

At each visit patients are asked regarding frequency and quality of sexual intercourse, ability to achieve orgasm and regrets about the procedure. A reference e-mail address is left to each patient where they can send any photo, question or doubt regarding the surgery and its follow-up (Figures 6, 7).

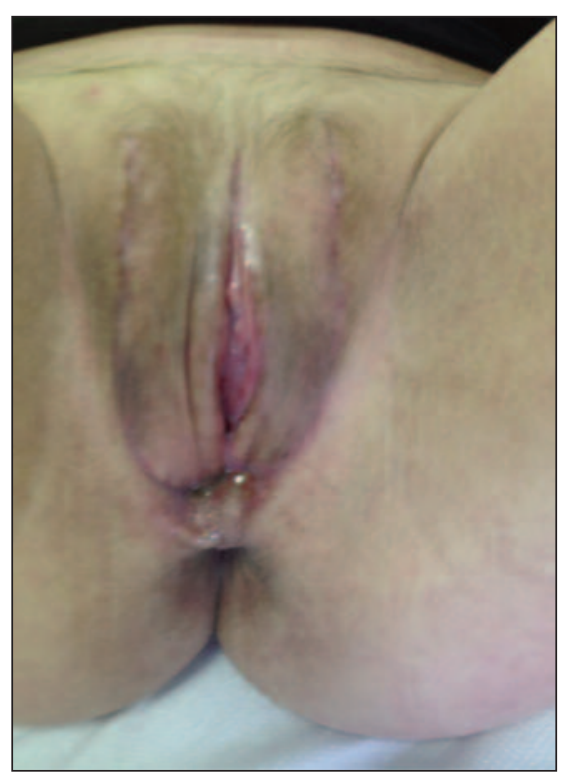

Figure 6.

6 months long-term follow-up.

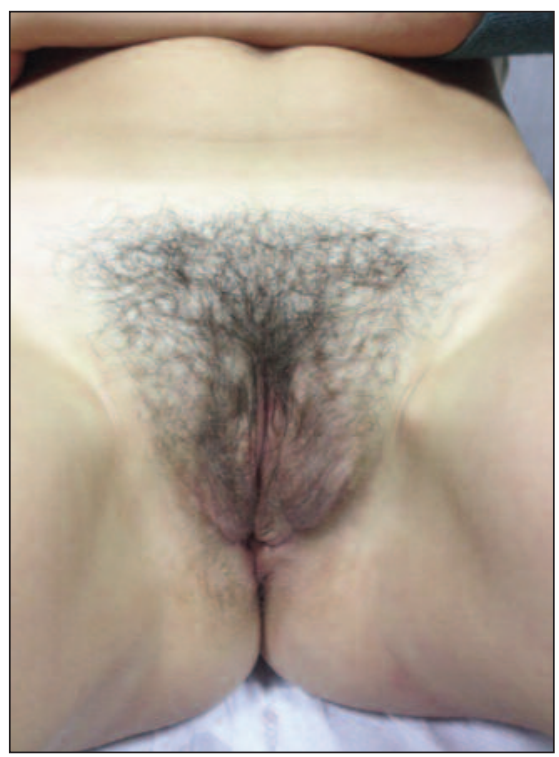

Figure 7.

Over 1-year long-term follow-up 


\section{RESULTS}

From February 2011 to April 2018, 94 transsexual women underwent MtoF gender affirming surgery. Mean age was 29,5 years (IQR: 24.75-34 months). Mean follow-up was 27.57 months (IQR: 25.77-29.45).

All patients were taking feminizing hormonal therapy with cyproterone acetate and estradiol for at least 3 years before surgery. Complications are reported in Table 1. During the 7 postoperative days 4 (4.25\%) patients required hematic transfusion. Partial necrosis of the labia minora occurred in $5(5.3 \%)$ patients but any of them required surgical revision. Vaginal stenosis occurred in 6 (6.38\%) patients who did not follow the daily vaginal dilatations program because complained uncomfortable filing during the procedure. Urethral meatus stricture occurred in $1(1.1 \%)$ patient. One $(1.1 \%)$ patient developed a rectovaginal fistula and protective colostomy was necessary. One (1.1\%) required 10 days of antibiotic therapy for a postoperative infectious complication.

Two patients underwent surgical revision for hematoma and edema of the lower limbs. At median follow-up of 27 months, 81 (86.1\%) patients reported vaginal intercourse and $78(82.9 \%)$ patients referred presence of erogenous sensitivity during dilatations, intercourse or masturbations.

\section{Discussion}

The provision of erogenous and tactile sensitivities of the neoclitoris in gender affirming surgery was first described by Brown (4) and Wesser (5), and this technique is used by most surgeons (6-12). However, most surgeons give the neoclitoris an oval or a triangular shape by excising a part of the glans and "wasting" erogenous tissue (13). In literature Dr. Preecha and his team (14) have developed a surgical technique where a good part of the glans is preserved by giving it an M-shape and its lateral flaps are used to form the labia minora. For the incision, they draw an M-shape marking on the dorsal glans by dermographic pen. Nevertheless, they excise the tissue included below the M-shape marking. Our approach differs from Preecha's because we draw a Yshape marking on the dorsal glans. Then we incise following the markings. At the end we obtain an M-shape

\section{Table 1.}

Postoperative complications in the period 2011-2018 $\left(n^{\circ}\right.$ tot patients $\left.=94\right)$.

\begin{tabular}{|ll|}
\hline Complication & $\mathbf{N}^{\circ}(\%)$ \\
\hline Bleeding & $4(4.25)$ \\
\hline Asymmetries & $3(3.19)$ \\
\hline Vaginal stenosis & $6(6.38)$ \\
\hline Urethral stenosis & $1(1.06)$ \\
\hline Recto-Vagina fistula & $1(1.06)$ \\
\hline Hypoesthesia of lower limb & $4(4.25)$ \\
\hline Hematoma of lower limb & $1(1.06)$ \\
\hline Edema of lower limb & $1(1.06)$ \\
\hline Urethral dolor & $1(1.06)$ \\
\hline Infection & $1(1.06)$ \\
\hline
\end{tabular}

glans spreading it after the incision. The central part of the M-shape glans forms the triangular neoclitoris meanwhile the lateral flaps are incised transversally for elongating themselves and form the labia minora. In this manner we obtain aesthetically pleasing genitalia esterna. With this technique cosmetic outcome and erogenous sensitivity are improved. Wagner et al. reported in a prospective trial the outcomes of sexual functions of 50 patients that underwent MtoF sex reassignment surgery. In this study 30\% patients did not reported presence of clitoral orgasm (15). Lawrence et al. analyzed retrospectively 232 patients, he reported that $15 \%$ of patients rarely refers presence of orgasm during masturbations while 18\% never experienced an orgasm (16). In both studies approximately $30 \%$ of patients reported insufficient orgasmic function. Our technique of neoclitoridolabioplasty could be considered a value option to reduce the risk of insufficient erogenous sensitivity. Some authors should advocate that hypersensitivity represent a commonly reported issue that routinely leads to a secondary surgical procedure to cover the clitoris with a hood flaps in order to protect it from an over stimulation (12). Remarkably discomfort due to an excessive sensitivity of the neoclitoris could be easily correct while lack of sensitivity is a not corrigible condition.

Finally, good erogenous sensitivity may permit more pleasant and comfortable dilatations that are essential to preserve the depth of the neovagina and patients particularly enjoyed the idea to preserve as much erogenous tissue as possible.

\section{ETHICAL CONSIDERATIONS}

The present study was designed in accordance with the ethical principles of the Declaration of Helsinki.

Considering the retrospective design, the study did not require approval by the local ethics committee and informed consent was not required because all procedures were performed according to the Standards of Care for the Health of Transsexual, Transgender, and GenderNonconforming People, Version 7.

\section{Conclusions}

Many procedures to reconfigure the glans in a neo-clitoris have been proposed and are currently used but any has been proven to be superior in terms of aesthetically and functional outcomes. Remarkably in some series up to $30 \%$ of patients refer inability to achieve orgasm due to an insufficient neoclitoris sensitivity. M-shape neoclitorolabioplasty represent a feasible alternative to the technique commonly used that permits the preservation of all the glans erogenous sensitivity.

\section{REFERENCES}

1. Association AP. Diagnostic and Statistical Manual of Mental Disorders (DSM-5®): American Psychiatric Publishing; 2013.

2. Organization WH. International Classification of Diseases, Tenth Edition.

3. Coleman E, Bockting W, Botzer M, et al. Standards of Care 
for the Health of Transsexual, Transgender, and GenderNonconforming People, Version 7. International Journal of Transgenderism. 2012; 13:165-232.

4. Brown J. Creation of a functional clitoris and aesthetically pleasing introitus in sex conversion. Transactions of the sixth international congress of plastic and reconstructive surgery Paris: Masson; 1976; p. 5.

5. Wesser DR. A single stage operative technique for castration, vaginal construction and perineoplasty in transsexuals. Arch Sex Behav. 1978; 7:309-23.

6. Selvaggi $G$, Monstrey S, Ceulemans $P$, et al. Genital sensitivity after sex reassignment surgery in transsexual patients. Ann Plas Surg. 2007; 58:427-33.

7. Fang RH, Chen CF, Ma S. A new method for clitoroplasty in male-to-female sex reassignment surgery. Plast Reconstr Surg. 1992; 89:679-82.

8. Eldh J. Construction of a neovagina with preservation of the glans penis as a clitoris in male transsexuals. Plast Reconstr Surg. 1993; 91:895-900.

9. Rubin SO. Sex-reassignment surgery male-to-female. Review, own results and report of a new technique using the glans penis as a pseudoclitoris. Scand J Urol Nephrol Suppl 1993; 154:1-28.
10. Rehman J, Melman A. Formation of neoclitoris from glans penis by reduction glansplasty with preservation of neurovascular bundle in male-to-female gender surgery: functional and cosmetic outcome. J Urol. 1999; 161:200-6.

11. Hage JJ, Karim RB. Sensate pedicled neoclitoroplasty for male transsexuals: Amsterdam experience in the first 60 patients. Annal Plas Surg. 1996; 36:621-4.

12. Sigurjonsson H, Mollermark C, Rinder J, et al. Long-Term Sensitivity and Patient-Reported Functionality of the Neoclitoris After Gender Reassignment Surgery. J Sex Med. 2017; 14:269-73.

13. Trombetta C, Liguori G, Benvenuto S, et al. [Neo-urethroclitoroplasty according to Petrovic]. Urologia 2011; 78:267-73.

14. Wangjiraniran B, Selvaggi $G$, Chokrungvaranont $P$, et al. Maleto-female vaginoplasty: Preecha's surgical technique. J Plast Surg Hand Surg. 2015; 49:153-9.

15. Wagner S, Greco F, Hoda MR, et al. Male-to-female transsexualism: technique, results and 3-year follow-up in 50 patients. Urol Int. 2010; 84:330-3.

16. Lawrence AA. Patient-reported complications and functional outcomes of male-to-female sex reassignment surgery. Arch Sex Behav. 2006; 35:717-27.

\section{Correspondence}

Andrea Cocci, MD, Ph.D. (Corresponding Author)

cocci.andrea@gmail.com

Augusto Delle Rose, MD

augustodellerose@libero.it

Marco Carini, MD, PhD

carini@unifi.it

Andrea Minervini, MD, PhD

Careggi Hospital, Department of Urology, University of Florence

Largo Brambilla, 3 - 50139, Florence (Italy)

Francesco Rosi, MD

francescorosi@gmail.com

Davide Frediani, MD

davide.frediani@gmail.com

Girolamo Morelli, MD

girolamomorelli@gmail.com

Department of Urology, University of Pisa, Pisa (Italy)

Michele Rizzo, MD

mik.rizzo@gmail.com

Carlo Trombetta, MF, PhD

Francesca Vedovo, MD

Department of Urology, University of Trieste, Trieste (Italy)

Gianmartin Cito, MD

gianmartin.cito@gmail.com

Simone Caroassai Grisanti, MD

simonecaroassai@libero.it

Valeria Matteucci, MD

Piero Buccianti, MD

Cristina Ceccarelli, MD

Department of General Surgery, University of Pisa, Pisa (Italy) 